\title{
Performance Analysis and Caching Design in Fog Radio Access Networks
}

\author{
Aoao Peng ${ }^{1,2,3}$, Yanxiang Jiang ${ }^{1,2,3, *}$, Mehdi Bennis ${ }^{4}$, Fu-Chun Zheng ${ }^{1,5}$, and Xiaohu You ${ }^{1}$ \\ ${ }^{1}$ National Mobile Communications Research Laboratory, Southeast University, Nanjing 210096, China \\ ${ }^{2}$ State Key Laboratory of Integrated Services Networks, Xidian University, Xi'an 710071, China \\ ${ }^{3}$ Key Laboratory of Wireless Sensor Network \& Communication, Shanghai Institute of Microsystem and \\ Information Technology, Chinese Academy of Sciences, 865 Changning Road, Shanghai 200050, China \\ ${ }^{4}$ Centre for Wireless Communications, University of Oulu, Oulu 90014, Finland \\ ${ }^{5}$ School of Electronic and Information Engineering, Harbin Institute of Technology, Shenzhen 518055, China \\ *E-mail: yxjiang@ @eu.edu.cn
}

\begin{abstract}
In this paper, the edge caching problem in fog radio access networks (F-RAN) is investigated. Considering that fog access points (F-APs) can offer cooperation gain by jointly transmitting the same file or content diversity gain by concurrently transmitting the coded subfiles, we propose to use both joint transmission (JT) strategy and parallel transmission (PT) strategy to serve users. Using stochastic geometry, we first derive the successful transmission probability (STP) with different transmission strategies, and further derive the fractional offloaded traffic (FOT). Finally, the optimal caching design is obtained by maximizing the STP and FOT. Simulation results show that our proposed caching design achieves a significant performance gain in comparison with the baselines.

Index Terms-Fog radio access networks, edge caching, stochastic geometry, successful transmission probability, fractional offloaded traffic.
\end{abstract}

\section{INTRODUCTION}

With the rapid proliferation of smart mobile devices and social networking services, wireless networks are under increased data traffic pressure. Fog radio access networks (F-RAN) can effectively alleviate network congestion by placing popular contents closer to users at off-peak hours [1]. Consequently, the fronthaul data traffic can be greatly reduced since there is no need to retrieve these cached contents from the cloud server. In the F-RAN, fog access points (F-APs) are equipped with limited storage space. Due to storage constraints, performance analysis and caching design are becoming ever more important in order to achieve a better network performance.

To obtain a tractable model, most of the existing literature utilizes stochastic geometry to model and analyze the network. The coverage probability and average achievable rate of the typical user were analyzed in [2] without considering content caching. In [3], the authors showed that content caching improves the system performance significantly, especially in limited-backhaul scenarios. In [4] and [5], the authors considered caching the most popular contents (MPC) at each small base station (SBS) or user equipment. However, the MPC caching design cannot provide content diversity gain.
If users are served by more than one SBS, i.e., the SBSs have overlapping coverage areas, the duplicate contents at each SBS may lead to low cache hit rate. In [6], a combined MPC and "largest content diversity" (LCD) caching design was applied in the clustercentric small cell networks. The less popular contents are equally partitioned and concurrently transmitted to increase content diversity gain, and successive interference cancellation (SIC) is employed to detect the uncoded subfiles. However, the uncoded caching design may not make full use of storage resources while the equal partition strategy may not achieve the optimal successful transmission probability (STP). In [7], the partition-based coded caching design was considered, where the optimal STP was derived by optimizing the number of subfiles that each file can be partitioned into. The authors in [8] proposed a similar core idea and analyzed the fractional offloaded traffic (FOT). In [7] and [8], it was assumed that the users have the SIC capability to detect the coded subfiles. However, when the file is not partitioned, the users are only served by the nearest SBS although all the SBSs have cached this file, i.e., cooperative transmission strategy is not considered.

Motivated by the aforementioned discussions, we propose a new caching design by considering both coded caching and cooperative transmission in the F-RAN. When the file is not partitioned, the joint transmission (JT) strategy is used to transmit the same cached file to achieve more cooperation gain. When the file is partitioned and encoded, the parallel transmission (PT) strategy is used to achieve more content diversity gain. The STP and FOT are analyzed and utilized to characterize the network performance. Then, the caching and transmitting strategy of each file is determined to obtain the maximum STP and FOT. Our proposed caching design can not only efficiently balance the tradeoff between cooperation gain and content diversity gain, but also fully exploit the storage resources.

The rest of this paper is organized as follows. In Section II, the system model is described. The performance analysis and caching design are presented in Section 


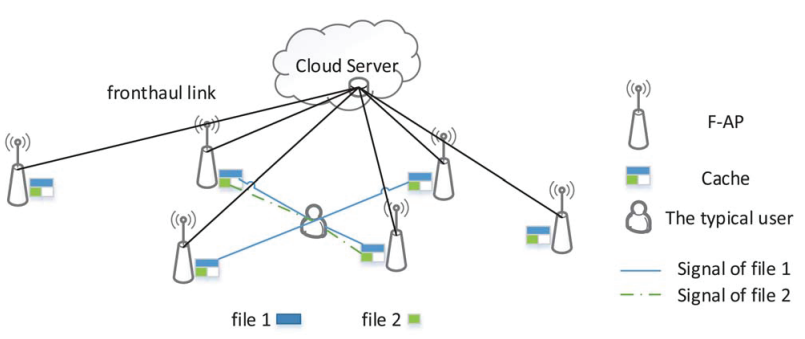

Fig. 1: Illustration of the F-RAN.

III. Simulation results are shown in Section IV. Final conclusions are drawn in Section V.

\section{SySTEM MOdel}

Consider the F-RAN as illustrated in Fig. 1, where the locations of the F-APs are modeled as homogeneous Poisson point process (PPP) distribution $\Phi_{\mathrm{F}}$ with given density $\lambda$. In the F-RAN, each F-AP has one transmit antenna and each user has one receive antenna. Both pathloss and small-scale Rayleigh fading are considered. For the pathloss, assume a standard distance-dependent power law attenuation, i.e., $r^{-\alpha}$ with $\alpha>2$, where $r$ denotes the distance from the considered F-AP to the typical user and $\alpha$ denotes the pathloss exponent. For the small-scale Rayleigh fading, it is modeled as an independent and identically exponential distribution with unit mean for all the links between F-APs and users. Besides, co-channel deployment is considered in this paper.

Let $N$ denote the size of the content library, and $\mathcal{N} \triangleq\{1,2, \cdots, N\}$ denote the index set of the files in the content library. For ease of analysis, assume that each file has the same size of $S$ bits [6]-[8], ${ }^{1}$ and the content popularity follows the Zipf distribution, i.e., $\tilde{p}_{n}=n^{-\gamma} / \sum_{\tilde{n}=1}^{N} \tilde{n}^{-\gamma}$, where the parameter $\gamma$ denotes the skewness of the distribution with a positive value. Assume that the cloud server contains all the files in the content library, while each F-AP is equipped with limited storage space that can store up to $C$ files. The content caching process includes two phases: content placement and content delivery, which will be presented below.

In the content placement phase, the F-APs fetch the corresponding contents from the cloud server through fronthaul links according to the caching design. ${ }^{2}$ Due to the limited SIC capability $D$ of the typical user, a file can only be partitioned into at most $D$ equal-sized subfiles. Let $s=\left[s_{1}, s_{2}, \cdots, s_{n}, \cdots, s_{N}\right]^{\mathrm{T}}$ denote the cache status vector of all the files in the library, where $s_{n} \in\{0,1\} \cup \overline{\mathcal{D}}$ denotes the cache status of file $n$ with $\overline{\mathcal{D}} \triangleq\{1 / 2,1 / 3, \cdots, 1 / D\}$. If $s_{n}=0$, file $n$ will not be cached in any F-AP, thus it has to be fetched from the cloud server through fronthaul links. If $s_{n}=1$, file

\footnotetext{
${ }^{1}$ Note that files with different sizes can always be partitioned into data packets with the same size.

${ }^{2}$ Note that the content placement phase generally occurs at off-peak hours and it does not occupy transmission time.
}

$n$ will be wholly cached at each F-AP. If $s_{n} \in \overline{\mathcal{D}}$, file $n$ will be partitioned into $1 / s_{n}$ subfiles and cached at the nearest $1 / s_{n}$ F-APs around the typical user. To fully exploit the caching capability, either maximum distance separable (MDS) codes or random linear network coding (RLNC) can be used to encode these subfiles and file $n$ can then be recovered from all its coded subfiles [8]. Note that each of the $1 / s_{n}$ nearest F-APs caches one of the coded subfiles with size $s_{n} S$ bits.

In the content delivery phase, according to whether the cached file is partitioned or not and the number of subfiles that it is partitioned into, different transmission strategies are designed to deliver this cached file. Consider the following two cooperative transmission strategies:

1) Joint Transmission (JT): If $s_{n}=1$, all the considered F-APs cache this file, e.g., file 1 as illustrated in Fig. 1. Assume that the $D$ nearest F-APs jointly transmit this file to increase the signal-to-interference ratio (SIR), thus cooperation gain can be obtained.

2) Parallel Transmission (PT): If $s_{n} \in \overline{\mathcal{D}}$, the $1 / s_{n}$ nearest F-APs concurrently transmit the $1 / s_{n}$ coded subfiles over the whole bandwidth, e.g., file 2 as illustrated in Fig. 1. At the receiver side, the typical user employs SIC to detect the $1 / s_{n}$ coded subfiles based on the distance order [9], thus content diversity gain can be obtained.

The objective of this paper is to find the optimal caching design $s$ by analyzing and maximizing the STP and FOT with limited storage space $C$ at each F-AP. ${ }^{3}$

\section{Performance Analysis and Caching DESIGN}

In this section, two metrics are considered for performance analysis and caching design. Firstly, the STP of the JT strategy is analyzed. Secondly, the STP of the PT strategy is presented. Based on the obtained STP, the closed-form expression of FOT is derived. Finally, the optimal caching design is obtained by maximizing the overall STP and FOT.

\section{A. Analysis of the STP}

According to [6], a successful delivery event occurs if a file can be delivered using bandwidth $W$ within transmission time $T$. Without loss of generality, focus on the analysis of the typical user within transmission time $T$. Let $\tau_{1}$ and $\tau_{2}$ denote the target SIRs for the JT and PT strategies, respectively. Let $p_{n}^{\mathrm{JT}}\left(\tau_{1}\right)$ and $p_{n}^{\mathrm{PT}}\left(\tau_{2}\right)$ denote the STP of the JT and PT strategies for file $n$, respectively. According to the total probability theorem, the STP over all the files in the content library can be

\footnotetext{
${ }^{3}$ When $s$ takes some specific value, the caching design degenerates to a deterministic and identical one. If $s_{n}=1$ for $1 \leq n \leq C$, the caching design degenerates to the MPC. If $s_{n}=1 / D$ for $1 \leq n \leq$ $D C \leq N$, it degenerates to the LCD.
} 
obtained as follows: ${ }^{4}$

$$
\begin{aligned}
& p=\sum_{n \in \mathcal{N}} \tilde{p}_{n}\left[p_{n}^{\mathrm{JT}}\left(\tau_{1}\right) 1\left(s_{n}=1\right)+\right. \\
&\left.p_{n}^{\mathrm{PT}}\left(\tau_{2}\right) 1\left(s_{n} \in \overline{\mathcal{D}}\right)\right],
\end{aligned}
$$

where $1(\cdot)$ denotes the indicator function.

1) JT Strategy: When file $n$ is wholly cached at each F-AP with $s_{n}=1$, it will be transmitted by using the JT strategy. The $D$ F-APs jointly transmit the same file to the typical user, and the received signals from the $D$ F-APs will be superimposed into a single stream to increase the SIR. Let $\boldsymbol{r}=\left[r_{1}, r_{2}, \cdots, r_{d}, \cdots, r_{D}\right]^{\mathrm{T}}$ denote the distance vector with the increasing distance order, where $r_{d}$ denotes the distance from the $d$ th nearest F-AP to the typical user with $d \in \mathcal{D} \triangleq\{1,2, \cdots, D\}$. By neglecting the background thermal noise, the SIR at the typical user can be expressed as follows:

$$
\mathrm{SIR}^{\mathrm{JT}}=\frac{\left|\sum_{d \in \mathcal{D}} h_{d} r_{d}^{-\alpha / 2}\right|^{2}}{\sum_{d \in \Phi_{\mathrm{F}} \backslash \mathcal{D}}\left|h_{d}\right|^{2} r_{d}^{-\alpha}},
$$

where $h_{d}$ denotes the Rayleigh fading channel coefficient between the $d$ th nearest F-AP and the typical user. Define the STP in this case as follows:

$$
p_{n}^{\mathrm{JT}}\left(\tau_{1}\right)=\mathrm{P}\left(\mathrm{SIR}^{\mathrm{JT}} \geq \tau_{1}\right) .
$$

Then, we have the following theorem.

Theorem 1: For the JT strategy, when the typical user requests file $n$ with the target SIR $\tau_{1}$, the STP can be expressed as follows:

$$
\begin{aligned}
& p_{n}^{\mathrm{JT}}\left(\tau_{1}\right)= \\
& \begin{cases}{\left[1+\rho\left(\tau_{1}, \alpha\right)\right]^{-1},} & D=1, \\
\int_{0}^{1} \cdots \int_{0}^{1}\left({ }_{2} F_{1}\right)^{-D} \mathrm{~d} u_{1} \cdots \mathrm{d} u_{D-1}, & D \geq 2,\end{cases}
\end{aligned}
$$

where

$$
\begin{aligned}
& \rho\left(\tau_{1}, \alpha\right)= \\
& \quad 2 \tau_{1}^{2 / \alpha} \alpha^{-1} B\left[2 / \alpha, 1-2 / \alpha,\left(1+\tau_{1}\right)^{-1}\right], \\
& { }_{2} F_{1}= \\
& \quad{ }_{2} F_{1}\left(-\frac{2}{\alpha}, 1 ; 1-\frac{2}{\alpha} ;-\frac{\tau_{1}}{1+\sum_{d=1}^{D-1} u_{d}^{-\alpha / 2}}\right),
\end{aligned}
$$

and $B(\cdot)$ and ${ }_{2} F_{1}(\cdot)$ denote the complementary incomplete beta function [10] and Gaussian hypergeometric function [11], respectively.

Proof: When $D=1$, the typical user associates with the nearest F-AP, and the STP can be readily

\footnotetext{
${ }^{4}$ Similar to [7] and [8], the service of uncached files is not investigated here. By exploiting the similar service mechanisms for uncached files in [6], the work in this paper can be easily extended. For uncached files, the fronthaul delay $T_{f}$ should be considered, then the maximum transmission time will be $\tilde{T}=T-T_{f}$. In this case, uncached files should be fetched from the cloud server to the nearest $D$ F-APs, and jointly transmitted to the typical user. Let $\tau_{3}=2^{S / W / \tilde{T}}-1$. Then, the STP in this case will be $p_{n}^{\mathrm{JT}}\left(\tau_{3}\right)$.
}

obtained according to [2].

When $D \geq 2$, from (2) and (3), we can obtain:

$$
\begin{aligned}
& p_{n}^{\mathrm{JT}}\left(\tau_{1}\right)= \\
& \quad \mathrm{P}\left(\left|\sum_{d \in \mathcal{D}} h_{d} r_{d}^{-\alpha / 2}\right|^{2} \geq \tau_{1} \sum_{d \in \Phi_{\mathrm{F}} \backslash \mathcal{D}}\left|h_{d}\right|^{2} r_{d}^{-\alpha}\right) .
\end{aligned}
$$

According to [6], we can obtain: $\left|\sum_{d \in \mathcal{D}} h_{d} r_{d}^{-\alpha / 2}\right|^{2} \sim$ $\exp \left(1 / \sum_{d \in \mathcal{D}} r_{d}^{-\alpha}\right)$. Correspondingly, (7) can be further expressed as follows:

$$
\begin{aligned}
p_{n}^{\mathrm{JT}}\left(\tau_{1}\right)= & \mathrm{E}_{\boldsymbol{r}}\left\{L_{\mathrm{I}}^{\text {out }}\left[\tau_{1}\left(\sum_{d \in \mathcal{D}} r_{d}^{-\alpha}\right)^{-1}\right] \mid \boldsymbol{r}\right\} \\
= & \int_{0}^{\infty} \int_{0}^{\tilde{r}_{D}} \cdots \int_{0}^{\tilde{r}_{D}} L_{\mathrm{I}}^{\text {out }}\left[\frac{\tau_{1}}{\sum_{d \in \mathcal{D}} \tilde{r}_{d}^{-\alpha}}\right] \\
& \times f_{\boldsymbol{r}}\left(\tilde{r}_{1}, \tilde{r}_{2}, \cdots, \tilde{r}_{D}\right) \mathrm{d} \tilde{r}_{D} \mathrm{~d} \tilde{r}_{D-1} \cdots \mathrm{d} \tilde{r}_{1},
\end{aligned}
$$

where $L_{\mathrm{I}}^{\text {out }}(\cdot)$ denotes the Laplace transform of the interference outside the circular area with radius $\tilde{r}_{D}$, and $f_{\boldsymbol{r}}\left(\tilde{r}_{1}, \tilde{r}_{2}, \cdots, \tilde{r}_{D}\right)$ denotes the joint probability density function (pdf) of $\boldsymbol{r}$.

For description convenience, define

$$
s=\tau_{1}\left(\sum_{d \in \mathcal{D}} \tilde{r}_{d}^{-\alpha}\right)^{-1} .
$$

Then, by exploiting the probability generating functional (PGFL) of PPP, the following relationship can be obtained:

$$
\begin{aligned}
& L_{\mathrm{I}}^{\text {out }}(s)=\mathrm{E}\left[\exp \left(-s \sum_{d \in \Phi_{\mathrm{F}} \backslash \mathcal{D}}\left|h_{d}\right|^{2} r_{d}^{-\alpha}\right)\right] \\
& =\exp \left\{-\pi \lambda \tilde{r}_{D}^{2}\left[{ }_{2} F_{1}\left(-\frac{2}{\alpha}, 1 ; 1-\frac{2}{\alpha} ;-\frac{s}{\tilde{r}_{D}^{\alpha}}\right)-1\right]\right\} .
\end{aligned}
$$

Consider that the $D-1$ F-APs are independently and uniformly distributed in the circular area whose radius is $\tilde{r}_{D}$ with the typical user being located at the center. For ease of analysis, assume that the typical user is at the origin. Therefore, the distribution of the $D-1$ nearest F-APs approximately follows a binomial point process (BPP) distribution in the circular area with radius $\tilde{r}_{D}$. Let $f_{r_{d} \mid r_{D}}\left(\tilde{r}_{d} \mid \tilde{r}_{D}\right)$ denote the pdf of $r_{d}$ conditioned on $r_{D}$. Then, we have: $f_{r_{d} \mid r_{D}}\left(\tilde{r}_{d} \mid \tilde{r}_{D}\right) \simeq 2 \tilde{r}_{d} \tilde{r}_{D}^{-2}$, for $0 \leq$ $\widetilde{r}_{d} \leq \widetilde{r}_{D}$ and $d=1,2, \cdots, D-1$. Let $f_{r_{D}}\left(\tilde{r}_{D}\right)$ denote the pdf of $r_{D}$. From [12], we also have:

$$
f_{r_{D}}\left(\tilde{r}_{D}\right)=\frac{2 \pi^{D} \lambda^{D} \tilde{r}_{D}^{2 D-1}}{(D-1) !} e^{-\pi \lambda \tilde{r}_{D}^{2}}
$$

Correspondingly, the joint pdf of $\boldsymbol{r}$ can be approximately expressed as follows:

$$
f_{\boldsymbol{r}}\left(\tilde{r}_{1}, \tilde{r}_{2}, \cdots, \tilde{r}_{D}\right) \simeq \prod_{d=1}^{D-1} 2 \tilde{r}_{d} \tilde{r}_{D}^{-2} f_{r_{D}}\left(\tilde{r}_{D}\right) .
$$




$$
\begin{aligned}
& \Upsilon\left(s_{n}\right)=\mathrm{P}\left(\operatorname{SIR}_{1 / s_{n}}^{\mathrm{PT}} \geq \tau_{2}\right) \mathrm{P}\left(\bigcap_{d=1}^{1 / s_{n}-1} \operatorname{SIR}_{d}^{\mathrm{PT}} \geq \tau_{2}\right)+ \\
& \quad \sum_{d=1}^{1 / s_{n}}(d-1) s_{n}\left[1-\mathrm{P}\left(\operatorname{SIR}_{d}^{\mathrm{PT}} \geq \tau_{2} \mid \bigcap_{\tilde{d}=1}^{d-1} \operatorname{SIR}_{\tilde{d}}^{\mathrm{PT}} \geq \tau_{2}\right)\right] \mathrm{P}\left(\bigcap_{\tilde{d}=1}^{d-1} \operatorname{SIR}_{\tilde{d}}^{\mathrm{PT}} \geq \tau_{2}\right) .
\end{aligned}
$$

Substitute (10) and (12) into (8). Through proper mathematical manipulations, the STP of file $n$ for $D \geq 2$ can be readily obtained as shown in (4). This completes the proof.

2) PT Strategy: When file $n$ is partitioned into $1 / s_{n}$ subfiles with $s_{n} \in \overline{\mathcal{D}}$ and cached at the nearest $1 / s_{n}$ F-APs around the typical user, it will be transmitted by using the PT strategy. The SIR at the typical user from the $d$ th nearest F-AP can be expressed as follows:

$$
\operatorname{SIR}_{d}^{\mathrm{PT}}=\frac{\left|h_{d}\right|^{2} r_{d}^{-\alpha}}{\sum_{\tilde{d} \in \Phi_{\mathrm{F}} \backslash \mathcal{D}}\left|h_{\tilde{d}}\right|^{2} r_{\tilde{d}}^{-\alpha}} .
$$

The corresponding STP of the $d$ th coded subfile can then be expressed as follows [7] [8]:

$$
\begin{aligned}
& p_{d}^{\mathrm{PT}}= \\
& \left\{1+2 \tau_{2}^{2 / \alpha} \alpha^{-1} B\left[2 / \alpha, 1-2 / \alpha,\left(1+\tau_{2}\right)^{-1}\right]\right\}^{-d} .
\end{aligned}
$$

Assume that the transmit events $\left\{\operatorname{SIR}_{d}^{\mathrm{PT}} \geq \tau_{2}\right\}_{d=1}^{1 / s_{n}}$ are independent. ${ }^{5}$ Let $p_{n}^{\mathrm{PT}}\left(d, \tau_{2}\right)$ denote the $\mathrm{STP}$ of the previous $d$ coded subfiles for $1 \leq d \leq 1 / s_{n}$. Then, it can be calculated as follows:

$$
\begin{aligned}
& p_{n}^{\mathrm{PT}}\left(d, \tau_{2}\right)=\prod_{\tilde{d}=1}^{d} p_{\tilde{d}}^{\mathrm{PT}}=\left\{1+2 \tau_{2}^{2 / \alpha} \alpha^{-1}\right. \\
& \left.\quad \times B\left[2 / \alpha, 1-2 / \alpha,\left(1+\tau_{2}\right)^{-1}\right]\right\}^{-d(d+1) / 2} .
\end{aligned}
$$

Correspondingly, for the PT strategy, when the typical user requests file $n$ with the target SIR $\tau_{2}$, the STP of file $n$ can then be expressed as follows:

$$
p_{n}^{\mathrm{PT}}\left(\tau_{2}\right)=p_{n}^{\mathrm{PT}}\left(1 / s_{n}, \tau_{2}\right) .
$$

\section{B. Analysis of the FOT}

Let $\Upsilon\left(s_{n}\right)$ denote the FOT of file $n$, which indicates the fractional traffic that file $n$ can be offloaded from the fronthual link and successfully delivered by the F-APs. When file $n$ is uncached, i.e., $s_{n}=0, \Upsilon\left(s_{n}\right)=0$. When file $n$ is wholly cached, i.e., $s_{n}=1$, the corresponding FOT is the STP of the JT strategy, i.e., $\Upsilon\left(s_{n}\right)=p_{n}^{\mathrm{JT}}\left(\tau_{1}\right)$.

When file $n$ is partitioned into $1 / s_{n}$ subfiles, i.e., $s_{n} \in$ $\overline{\mathcal{D}}$, it will be transmitted by using the PT strategy. The typical user can detect the $d$ th coded subfile under the condition that all the coded subfiles from the $d-1$ nearer

\footnotetext{
${ }^{5}$ Here we follow the same assumption as [7]-[9], which makes the analysis more tractable.
}

F-APs have been detected successfully. Otherwise, only $(d-1) s_{n}$ fraction of file $n$ can be offloaded, and the other subfiles have to be retransmitted to recover the whole file. Then, we have the following theorem.

Theorem 2: Assume that the transmit events $\left\{\mathrm{SIR}_{d}^{\mathrm{PT}} \geq \tau_{2}\right\}_{d=1}^{1 / s_{n}}$ are independent. Then, the FOT of file $n$ can be expressed as follows:

$$
\begin{array}{r}
\Upsilon\left(s_{n}\right)= \\
\begin{cases}0, & s_{n}=0, \\
p_{n}^{\mathrm{JT}}\left(\tau_{1}\right), & s_{n}=1, \\
s_{n} \sum_{d=1}^{1 / s_{n}} p_{n}^{\mathrm{PT}}\left(d, \tau_{2}\right), & s_{n} \in \overline{\mathcal{D}} .\end{cases}
\end{array}
$$

Proof: It is easy to obtain the FOT of file $n$ when $s_{n}=0$ and $s_{n}=1$. When $s_{n} \in \overline{\mathcal{D}}$, according to the above analysis, the corresponding FOT can be calculated by (18) as shown at the top of this page, where the first term denotes the FOT when all the coded subfiles are detected successfully, and the second term denotes the FOT when only parts of the subfiles are detected successfully. According to the independent assumption concerning the transmit events, we can further obtain:

$$
\begin{aligned}
\Upsilon\left(s_{n}\right) & =\prod_{d=1}^{1 / s_{n}} p_{d}^{\mathrm{PT}}+\sum_{d=1}^{1 / s_{n}} s_{n}(d-1)\left(1-p_{d}^{\mathrm{PT}}\right) \prod_{\tilde{d}=1}^{d-1} p_{\tilde{d}}^{\mathrm{PT}} \\
& =s_{n} \sum_{d=1}^{1 / s_{n}} \prod_{\tilde{d}=1}^{d} p_{\tilde{d}}^{\mathrm{PT}} .
\end{aligned}
$$

Then, according to (15), the FOT of file $n$ for $s_{n} \in \overline{\mathcal{D}}$ can be expressed as follows:

$$
\Upsilon\left(s_{n}\right)=s_{n} \sum_{d=1}^{1 / s_{n}} p_{n}^{\mathrm{PT}}\left(d, \tau_{2}\right), s_{n} \in \overline{\mathcal{D}} .
$$

This completes the proof.

By averaging $\Upsilon\left(s_{n}\right)$ over all the files in the content library, the overall FOT $\Upsilon$ can then be calculated as follows:

$$
\Upsilon=\sum_{n=1}^{N} \tilde{p}_{n} \Upsilon\left(s_{n}\right) \text {. }
$$

\section{Caching Design}

According to [6], for the JT strategy, it can yield larger STP and cooperation gain for one file. However, since a whole file occupies larger storage space and fewer files can be cached in the storage space of one F-AP, smaller content diversity gain can be obtained. Whereas for the PT strategy, it can yield smaller STP and cooperation gain for one file. However, since a coded subfile occupies fewer storage space and more files can be cached in the storage space of one F-AP, larger content diversity 
gain can be obtained. To efficiently control the tradeoff between cooperation gain and content diversity gain, we propose to find the optimal caching design $s$ by maximizing the STP and FOT.

By taking the STP into account, the caching optimization problem can be formulated as follows:

$$
\begin{array}{ll} 
& \max _{s} p, \\
\text { s.t. } & s_{n} \in\{0,1\} \cup \overline{\mathcal{D}}, n \in \mathcal{N}, \\
& \sum_{n \in \mathcal{N}} s_{n} \leq C .
\end{array}
$$

Define $\tilde{\mathcal{D}}=\mathcal{D} \cup\{D+1\}$. Introduce an $N \times(D+1)$ matrix $\boldsymbol{X}=\left(x_{n, d}\right)_{n \in \mathcal{N}, d \in \tilde{\mathcal{D}}}$ with $x_{n, d} \in\{0,1\}$ indicating the cache status of file $n$. Let $p_{n, d}$ denote the STP of file $n$ with its corresponding transmission strategy. If $x_{n, 1}=$ 1 , file $n$ will be transmitted by using the JT strategy and $p_{n, 1}=p_{n}^{\mathrm{JT}}\left(\tau_{1}\right)$. If $x_{n, d}=1$ for $d=2,3, \cdots, D$, file $n$ will be partitioned into $d$ subfiles, transmitted by using the PT strategy, and $p_{n, d}=p_{n}^{\mathrm{PT}}\left(d, \tau_{2}\right)$. If $x_{n, D+1}=1$, file $n$ will not be cached and $p_{n, D+1}=0$. Correspondingly, the above optimization problem can be transformed into the following equivalent multiplechoice knapsack problem (MCKP):

$$
\begin{aligned}
& \max _{\boldsymbol{X}} \sum_{n \in \mathcal{N}} \tilde{p}_{n} \sum_{d \in \tilde{\mathcal{D}}} p_{n, d} x_{n, d}, \\
\text { s.t. } & x_{n, d} \in\{0,1\}, n \in \mathcal{N}, d \in \tilde{\mathcal{D}}, \\
& \sum_{d \in \tilde{\mathcal{D}}} x_{n, d}=1, n \in \mathcal{N}, \\
& \sum_{n \in \mathcal{N}} \sum_{d \in \mathcal{D}} d^{-1} x_{n, d} \leq C .
\end{aligned}
$$

The above $0-1$ integer programming problem can be solved by utilizing the INTLINPROG toolbox of MAT$\mathrm{LAB}$, and the optimal caching status matrix $\boldsymbol{X}$ as well as the optimal STP can then be obtained.

Similarly, we can formulate the optimization problem concerning the FOT and find the corresponding optimal caching design.

\section{Simulation Results}

In this section, we evaluate the performance of our proposed caching design via simulations. The simulation parameters are set as follows: the path loss parameter $\alpha=4$, the bandwidth $W=10 \mathrm{MHz}$, the content library size $N=1000$, the SIC capability $D=4$, and the transmission time $T=1 \mathrm{~ms}$. The MPC and LCD caching designs are chosen as the baselines. ${ }^{6}$

Impact of Cache Size C: In Fig. 2 (a) and (b), we show the STP and FOT of our proposed caching design and the two baselines with different cache size $C$. It can be observed that the performance of all the considered caching designs improves with the increase of cache

\footnotetext{
${ }^{6}$ For the PT strategy, when the files are partitioned into different subfiles, different number of F-APs are required to transmit them, which will consume different transmit power. Note that we do not consider energy efficiency but STP (FOT) in this paper. Therefore, with the same transmission time $T$, different caching designs, such as MPC and LCD, are comparable.
}

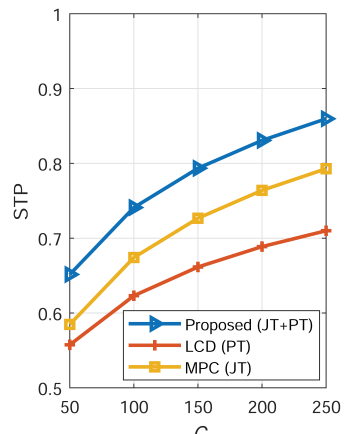

(a) STP vs. cache size with Zipf parameter $\gamma=1$

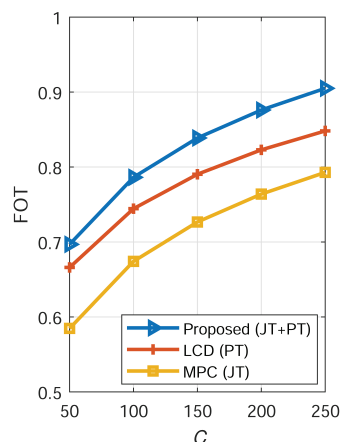

Fig. 2: The STP and FOT versus cache size $C$.

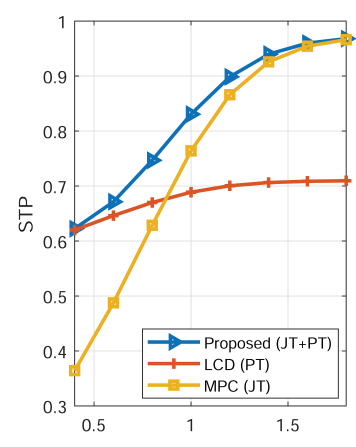

(a) STP vs. $\gamma$ with $C=200$

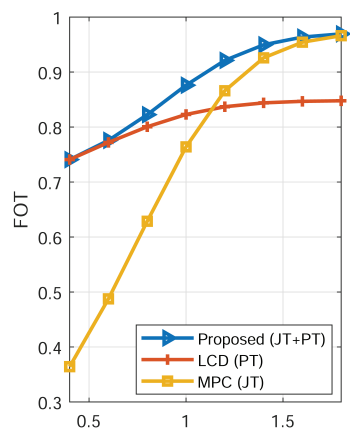

(b) FOT vs. $\gamma$ with $C=200$
Fig. 3: The STP and FOT versus Zipf parameter $\gamma$.

size. The reason is that larger cache size means that each F-AP can store more files and the probability that a file can be fetched locally becomes larger. It can also be observed that our proposed caching design is superior to the two baselines for all $C$. The reason is that under the condition of limited storage space, our proposed caching design can efficiently exploit the storage space by using both the JT and PT strategies whereas the MPC caching design only uses the JT strategy and the LCD caching design only uses the PT strategy.

Impact of Zipf Parameter $\gamma$ : In Fig. 3 (a) and (b), we show the STP and FOT of our proposed caching design and the two baselines with different Zipf parameter $\gamma$. It can be observed that our proposed caching design outperforms the two baselines for all $\gamma$. When $\gamma$ is large, most content requests are concentrated on a few popular contents, hence the MPC caching design can achieve larger gain and the proposed caching design gradually degenerates to the MPC. When $\gamma$ is small, the popularity distribution tends to be uniform, hence the LCD caching design can achieve larger gain and the proposed caching design gradually degenerates to the LCD. Our proposed caching design combines the characteristics of the MPC and LCD caching designs flexibly, and thus it can achieve a relatively better performance. 


\section{Conclusions}

In this paper, we have studied the performance analysis and caching design in the F-RAN. By using the theory of stochastic geometry, the joint transmission strategy for unpartitioned files was analyzed, then the parallel transmission strategy for partitioned files was analyzed. Finally, we found the optimal caching design by maximizing the STP and FOT. Simulation results showed that our proposed caching design can achieve a better performance than the baselines.

\section{ACKNOWLEDGMENTS}

This work was supported in part by the Natural Science Foundation of Jiangsu Province under grant BK20181264, the Hong Kong, Macao and Taiwan Science \& Technology Cooperation Program of China under grant 2014DFT10290, the Research Fund of the State Key Laboratory of Integrated Services Networks (Xidian University) under grant ISN19-10, the Research Fund of the Key Laboratory of Wireless Sensor Network $\&$ Communication (Shanghai Institute of Microsystem and Information Technology, Chinese Academy of Sciences) under grant 2017002, the National Basic Research Program of China (973 Program) under grant 2012CB316004, and the U.K. Engineering and Physical Sciences Research Council under Grant EP/K040685/2.

\section{REFERENCES}

[1] M. Peng, S. Yan, K. Zhang, and C. Wang, "Fog-computing-based radio access networks: Issues and challenges," IEEE Network, vol. 30, no. 4, pp. 46-53, July 2016.

[2] J. G. Andrews, F. Baccelli, and R. K. Ganti, "A tractable approach to coverage and rate in cellular networks," IEEE Trans. Commun., vol. 59, no. 11, pp. 3122-3134, Nov. 2011.

[3] E. Bastug, M. Bennis, and M. Debbah, "Living on the edge: The role of proactive caching in 5G wireless networks," IEEE Commun. Mag., vol. 52, no. 8, pp. 82-89, Aug. 2014.

[4] C. Yang, Y. Yao, Z. Chen, and B. Xia, "Analysis on cacheenabled wireless heterogeneous networks," IEEE Trans. Wireless Commun., vol. 15, no. 1, pp. 131-145, Jan. 2016.

[5] Z. Yan, S. Chen, Y. Ou, and H. Liu, "Energy efficiency analysis of cache-enabled two-tier HetNets under different spectrum deployment strategies," IEEE Access, vol. 5, pp. 6791-6800, 2017.

[6] Z. Chen, J. Lee, T. Q. S. Quek, and M. Kountouris, "Cooperative caching and transmission design in cluster-centric small cell networks," IEEE Trans. Wireless Commun., vol. 16, no. 5, pp. 3401-3415, May 2017.

[7] D. Jiang and Y. Cui, "Partition-based caching in large-scale SIC-enabled wireless networks," IEEE Trans. Wireless Commun., vol. 17, no. 3, pp. 1660-1675, Mar. 2018.

[8] X. Xu and M. Tao, "Modeling, analysis, and optimization of coded caching in small-cell networks," IEEE Trans. Commun. vol. 65, no. 8, pp. 3415-3428, Aug. 2017.

[9] M. Wildemeersch, T. Q. S. Quek, M. Kountouris, A. Rabbachin, and C. H. Slump, "Successive interference cancellation in heterogeneous networks," IEEE Trans. Commun., vol. 62, no. 12, pp. 4440-4453, Dec. 2014.

[10] H. E. Mckean, Tables of the Incomplete Beta Function. Technometrics, 1968.

[11] L. J. Slater, Generalized Hypergeometric Functions. Cambridge University Press, 1966.

[12] M. Haenggi, "On distances in uniformly random networks," IEEE Trans. Inf. Theory, vol. 51, no. 10, pp. 3584-3586, Oct. 2005. 